\title{
Women in the Middle: Cuba's Sandwich Generation
}

\section{By Leticia Artiles, PhD, MS}

I once met anthropologist Dolores Juliano, who wisely observed that women "don't live more, they just die later." Women's longer lifespan is almost mythical, but our longer lives are fraught with excessive morbidity. And extended life expectancy itself - a phenomenon that has caught health systems unprepared - has boosted the role of "the caregiver" needed for older persons and the chronically ill.

In Cuba, middle-aged women are at the epicenter of family dynamics; the majority of them still work, even though they may have reached retirement age; and they are the caregivers for children and aging parents alike. As they get older themselves, these women more than men have had to change their life plans, leave their work, and dedicate themselves entirely to people in their care, bearing the guilt that arises when tension and stress convert to irritability - usually towards the one in their care.

One woman, who took care of her Alzheimer-stricken mother, told me: "I feel terrible. l'm bathing my mother, using a soft sponge so I won't scratch her skin, and at the same time I'm yelling at her to stay still... but l'm at the end of my rope." Another said: "I feel as if my batteries were just running down."

This kind of erosion in women's perception of their own health isn't registered in the statistics; it doesn't show up in any system of epidemiological surveillance. Yet, reviewing Cuban health data for 2004-2005, I notice that the gap between mortality rates from heart disease is closing between men and women, the ratio of male to female deaths dangerously approaching 1:1.

From an epidemiological perspective, women are dying more often from this cause; from a gender perspective, women are dying more like men. Which leads to other nagging questions: what happened to the biological protection that women were supposed to enjoy against heart disease, at least until menopause? What's the story behind these new statistics?

And after the questions sound the alarm: the mortality rate from heart disease among women aged 50-64 is rising, despite the guarantees of a free, universal public health system like ours. Evidence is mounting that suggests women approaching menopause urgently need a medical-social diagnosis to look deeper into the small universe that surrounds each of them - family, work, partner, community - to discover those invisible processes in their daily lives that are tipping the balance towards disease and death. Simply put, we need a "gender-based epidemiological profile" to describe the contradictions that accompany the process of health and disease, reflecting the unequal burden borne by women and its influence on their health.

In a recent Cuban study, over 100 women age 40-59 were interviewed, identifying factors that increased stress levels in their daily lives. They cited tension and worries associated with caregiving for older, sick, or younger family members; the unequal burden of housework, which in some homes was beginning to be shared but not nearly equally, leaving the most onerous jobs at the woman's doorstep; and the role of family "mediator," navigating the complexities of intergenerational households.

The majority of these women worked outside the home, but the housework still fell on their shoulders, creating double and triple shifts, draining their energy day by day. Most of their jobs involved intellectual work, requiring after-work thought and planning, cutting into time usually dedicated to domestic chores. In general, the women referred to inequities in gender roles within the home, and to relations with their partners dominated by masculine models of affection and sexuality.

The women surveyed generally felt they received little recognition, and they blamed themselves for not achieving the functional stability expected by everyone around them, including themselves.

All this influenced their wellbeing: anxiety, depression, irritability, and other emotional changes, as well as vasomotor changes (primarily hot flashes and sweats) were more frequently perceived. The study revealed that gender-related overload at least doubled perception of menopausal symptoms, with direct repercussion in the quality of life for these women.

\section{Gender-related overload at least doubled perception of menopausal symptoms}

The most significant thing is that these phenomena aren't contemplated in general diagnoses, reserving them for mental health specialists. And when such a specialist does identify these symptoms, instead of associating them with social relations, they are explained solely in biological or psychiatric terms - which of course is easier.

What's needed is a "medication" that transforms society and its gender inequities. In fact, if we're going to involve gender in an analysis of health status, then we have to talk about the "epidemiology of gender differences".

In Cuba, work is ongoing in this direction, and we have the advantage that the Obstetrics and Gynecology Society has a section on Climaterium and Menopause, which for the last 15 years has offered comprehensive attention with a social approach. In 2006, the 2nd National Consensus on Climaterium and Menopause emphasized the use of a social-medical diagnosis as a methodological resource to actively incorporate women into their own health care. This comprehensive, individualized and genderbased focus constitutes an organizational innovation for Cuba and Latin America. Its impact thus far is captured in the words of one woman I met: "When I went for my appointment, I felt like a person, not just a uterus."

But more needs to be done. Much more. The statistics have yet to include "gender overload" as a risk factor for middle-aged women. Menopause still bears the blame for symptoms that offer broader clues to the deterioration of health. And then we have that pesky data showing that heart disease is catching up. $-1 /$ - 